\title{
Association of triglyceride-glucose index and stroke recurrence among nondiabetic patients with acute ischemic stroke
}

\author{
Xiaomeng Yang ${ }^{1,2 \dagger}$, Guangyao Wang ${ }^{3 \dagger}$, Jing Jing ${ }^{1,2}$, Anxin Wang ${ }^{1,2}$, Xiaoli Zhang ${ }^{1,2}$, Qian Jia ${ }^{1,2}$, Xia Meng $^{1,2}$,
} Xingquan Zhao ${ }^{1,2}$, Liping Liu ${ }^{1,2}$, Hao Li ${ }^{1,2}$, Yongjun Wang ${ }^{1,24^{*}}$ and Yilong Wang ${ }^{1,2^{*}}$

\begin{abstract}
Backgroud and purpose: Triglyceride-glucose (TyG) index has been considered a surrogate marker of insulin resistance. We investigated the association between TyG index and stroke recurrence and compared the effectiveness of TyG index with homeostasis model assessment of insulin resistance (HOMA-IR) in predicting stroke recurrence and death in nondiabetic acute ischemic stroke patients.

Methods: Nondiabetic acute ischemic stroke patients from the ACROSS-China (Abnormal Glucose Regulation in Patients with Acute Stroke across China) registry were included. TyG index was performed and classified into four groups by quartiles. The outcomes were stroke recurrence and death within 1 year. The association between TyG index and the risk of stroke recurrence and death were analyzed by Cox regression models. Receiver operating characteristic (ROC) curve analysis was performed to evaluate the prediction of TyG index and HOMA-IR for stroke recurrence and death. Delong test was used for comparing the differences between area under the curve (AUC) of TyG index and HOMA-IR.
\end{abstract}

Results: Among the 1226 patients included, the median (interquartile range) of TyG index was 5.8 (5.5-6.2). Both the third and fourth quartiles of TyG index were associated with an increased risk of stroke recurrence (adjusted hazard ratio 2.04, 95\% confidence interval 1.26-3.31; adjusted hazard ratio 1.86, 95\% confidence interval 1.13-3.06). Patients with fourth quartiles of TyG index were associated with a higher mortality risk (adjusted hazard ratio, 2.91; 95\% confidence interval, 1.62-2.53). Regarding stroke recurrence within 1 year, the AUC (95\% confidence interval) of the ROC curve for the TyG index was similar to that of the HOMA-IR[0.56 (0.52-0.61) vs $0.57(0.52-0.61) ; P=0.93]$. Regarding death within 1 year, the AUCs (95\% confidence interval) of the ROC curve for the TyG index and HOMA-IR were 0.55 $(0.50-0.61)$ and $0.59(0.53-0.64)$, respectively $(P=0.32)$.

Conclusions: Elevated TyG index was associated with an increased risk of stroke recurrence and death. However, neither of TyG nor HOMA-IR can be a qualified predictor of stroke recurrence and death in nondiabetic acute ischemic stroke patients.

Keywords: Insulin resistance, Ischemic stroke, Prognosis, Triglyceride-glucose index, stroke recurrence, death

\footnotetext{
*Correspondence: yongjunwang@ncrcnd.org.cn; yilong528@gmail.com ${ }^{+}$Xiaomeng Yang and Guangyao Wang contributed equally to this manuscript.

${ }^{1}$ Department of Neurology, Beijing Tiantan Hospital, Capital Medical University, No.119, South 4th Ring West Road, Fengtai District, Beijing 100070, China

Full list of author information is available at the end of the article
}

\begin{abstract}
Introduction
Insulin resistance was common in subjects with multiple metabolic disorders and stroke [1, 2]. Insulin resistance, measured by homeostasis model assessmentinsulin resistance (HOMA-IR) index, was associated with
\end{abstract}


an increased risk of stroke recurrence in nondiabetic patients with acute ischemic stroke [3]. Previous studies have illustrated the importance of triglycerides in the pathogenesis of insulin resistance and the biological plausibility for using triglycerides levels as a surrogate in the identification of insulin resistance. Besides, the levels of fasting triglyceride and glucose are universally available in the clinical practice $[4,5]$. Some studies showed that Triglyceride-glucose (TyG) index was more independently associated with the presence of coronary artery atherosclerosis [6], carotid atherosclerosis [7], and the development of diabetes [8] than HOMA-IR. Another study showed that TyG index was similar to HOMA-IR in prediction of diabetes [9]. However, few studies have investigated the relationship between TyG index and stroke recurrence or death and compared the effectiveness of TyG index and HOMA-IR in predicting stroke recurrence or death in nondiabetic acute ischemic stroke patients.

Using data from the ACROSS-China registry (Abnormal Glucose Regulation in Patients With Acute Stroke Across China), we aim to investigate the association between TyG index and stroke recurrence together with death, and compare effectiveness of TyG index and HOMA-IR in predicting stroke recurrence and death of nondiabetic acute ischemic stroke patients.

\section{Methods}

\section{Study participants}

The detailed design of the ACROSS-China study has been described previously [10]. Briefly, ACROSS-China is a nationwide prospective cohort study which aimed to investigate the prevalence of abnormal glucose regulation in hospitalized patients with a first-ever ischemic and hemorrhagic stroke within 14 days after onset and the relationship of abnormal glucose regulation with the prognosis of stroke from 2008 to 2009 across China. The ACROSS-China study has been approved by the ethics committee of Beijing Tiantan Hospital, Capital Medical University and all participating hospitals. All participants or their legal representatives have provided written informed consent.

Acute ischemic stroke patients without a history of diabetes mellitus in the ACROSS-China study were included in this analysis. The definition of history of diabetes mellitus was based on the previous medical records and use of hypoglycemia agents. According to the World Health Organization criteria [11], acute ischemic stroke was diagnosed with confirmation by brain computed tomography or magnetic resonance imaging.

\section{Data collection}

Patient information including demographic characteristics, medical history, and risk factors were recorded within $24 \mathrm{~h}$ after admission through face-to-face interviews by trained interviewers from the participating hospitals according to a standardized protocol. According to the TOAST (Trial of ORG 10,172 in Acute Stroke Treatment) criteria, the etiologic subtypes of acute ischemic stroke were classified [12]. The National Institutes of Health Stroke Scale (NIHSS) was used to assess the severity of neurological impairment within $24 \mathrm{~h}$ after admission. Complications of pulmonary or urinary infection and medications used during hospitalization were recorded.

\section{Evaluation of TyG index}

The first overnight fasting venous blood samples (at least $8 \mathrm{~h}$ ) were drawn to measure the fasting triglyceride. Triglyceride was enzymatically measured using spectrophotometric methods. Fasting venous blood samples were drawn to measure fasting glucose levels on day $14 \pm 3$ after stroke or before discharge (if length of stay in hospital was $<14$ days) after overnight fasting (at least $8 \mathrm{~h}$ ). Fasting glucose was measured by using an enzymatic method. TyG index was calculated as $\ln$ (fasting triglyceride $[\mathrm{mg} / \mathrm{dL}] \times$ fasting glucose $[\mathrm{mg} / \mathrm{dL}] / 2)[6]$.

\section{Evaluation of HOMA-IR}

Fasting venous blood samples were drawn to measure fasting insulin on day $14 \pm 3$ after stroke or before discharge (if length of stay in hospital was $<14$ days) after overnight fasting (at least $8 \mathrm{~h}$ ). Fasting insulin was measured by using a competitive radioimmunoassay (Diagnostic Products Corporation). The fasting glucose used to calculate the HOMA-IR was the same as the TyG index. HOMA-IR was calculated as fasting insulin $[\mu \mathrm{U} /$ $\mathrm{mL}] \times$ fasting glucose $[\mathrm{mmol} / \mathrm{L}] / 22.5)[13]$.

\section{Outcomes and follow-up}

All patients enrolled in the ACROSS-China study were followed up at 12 months after stroke onset by a centralized telephone follow-up, which was based on a shared standardized interview protocol. The outcomes were stroke recurrence and death within 1 year. Stroke recurrence was defined as an aggravated primary neurological deficit, a new neurological deficit, or re-hospitalization caused by ischemic or hemorrhagic stroke [14].

\section{Statistical analysis}

Patients were divided into four groups according to the quartiles of TyG index. Continuous variables were presented as median with interquartile range and categorical variables were presented as proportions. The baseline variables among different quartiles of TyG 
index were compared by Wilcoxon or Kruskal-Wallis test for continuous variables and $\mathrm{x} 2$ test for categorical variables. The associations of TyG index with stroke recurrence and death were analyzed by using Cox proportional-hazards regression models. In the multivariable model, all the potential confounders listed in Table 1 were included. The area under the curve (AUC) of the receiver operating characteristics (ROC) curve and a $95 \%$ confidence interval were calculated to compare the predictive power of the HOMA-IR and TyG index for stroke recurrence and death. Delong test was used for comparing the differences between AUCs. We further evaluated the associations between TyG index and risk of outcomes in nondiabetic acute ischemic stroke patients without cardioembolism.

\section{Results}

Study participants

A total of 2639 patients with acute ischemic stroke were enrolled in the ACROSS-China study. After excluding 534 patients with a history of diabetes mellitus, 568 patients with missing data on fasting insulin, fasting glucose and fasting triglyceride, 311 patients lost to follow up at 12-months, 1226 nondiabetic acute ischemic stroke patients were included in this analysis (Fig. 1). Baseline characteristics of nondiabetic acute ischemic stroke patients included and not included were almost balanced, except that the patients included in this analysis were more likely to have higher body mass index and higher proportion of taking anticoagulation during hospitalization (Supplemental Table 1).

Table 1 Characteristics of patients included according to TyG index quartiles

\begin{tabular}{|c|c|c|c|c|c|}
\hline \multirow[t]{2}{*}{ Characteristics } & \multicolumn{4}{|c|}{ Quartiles of the TyG index } & \multirow[t]{2}{*}{$P$ value } \\
\hline & $\begin{array}{l}\text { Quartiles 1, } \\
4.31-5.48\end{array}$ & $\begin{array}{l}\text { Quartiles 2, } \\
5.48-5.81\end{array}$ & $\begin{array}{l}\text { Quartiles 3, } \\
5.81-6.22\end{array}$ & $\begin{array}{l}\text { Quartiles 4, } \\
6.22-8.17\end{array}$ & \\
\hline Patients, n (\%) & 306 & 307 & 307 & 306 & \\
\hline Male, n (\%) & 197(64.8) & 182(59.5) & $191(62.2)$ & $202(66.7)$ & 0.28 \\
\hline Age, y, median (IQR) & $67.0(55.0-75.0)$ & $65.0(53.0-75.0)$ & $60.0(53.0-69.0)$ & $59.0(51.0-70.0)$ & $<0.001$ \\
\hline BMI, median (IQR) & $24.0(21.6-26.2)$ & $24.9(22.5-27.0)$ & $24.9(22.9-27.3)$ & $25.2(23.4-27.4)$ & $<0.001$ \\
\hline Smoking, n (\%) & & & & & 0.49 \\
\hline Current smoker & $99(32.4)$ & $93(30.3)$ & $99(32.2)$ & 114(37.3) & \\
\hline Ever smoker & $31(10.1)$ & $26(8.5)$ & $34(11.1)$ & $30(9.8)$ & \\
\hline Nonsmoker & $176(57.5)$ & 188(61.2) & 174(56.7) & $162(52.9)$ & \\
\hline \multicolumn{6}{|l|}{ Medical history } \\
\hline Hypertension, n (\%) & $161(52.6)$ & $174(56.7)$ & 198(64.5) & 203(66.3) & 0.001 \\
\hline Hyperlipidemia, n (\%) & $20(6.5)$ & $28(9.1)$ & $43(14.0)$ & $50(16.3)$ & $<0.001$ \\
\hline Atrial fibrillation, n (\%) & $34(11.1)$ & $17(5.5)$ & $14(4.6)$ & 10(3.3) & $<0.001$ \\
\hline Coronary heart disease, n (\%) & $32(10.5)$ & $39(12.7)$ & $39(12.7)$ & $38(12.4)$ & 0.80 \\
\hline \multicolumn{6}{|l|}{ Medication during hospitalization, $\mathrm{n}(\%)$} \\
\hline Antihypertensive drugs & 118(38.6) & $136(44.3)$ & 145(47.2) & 144(47.1) & 0.11 \\
\hline Statins & $156(51.0)$ & 138(45.0) & $157(51.1)$ & $176(57.5)$ & 0.02 \\
\hline Intravenous alteplase & $8(2.6)$ & $7(2.3)$ & $9(2.9)$ & 12(3.9) & 0.66 \\
\hline Antiplatelet & 194(63.4) & 187(60.9) & 188(61.2) & 194(63.4) & 0.87 \\
\hline Anticoagulation & $25(8.2)$ & $22(7.2)$ & $15(4.9)$ & $18(5.9)$ & 0.37 \\
\hline \multicolumn{6}{|c|}{ Complications during hospitalization, n (\%) } \\
\hline Pulmonary infection & $29(9.5)$ & $36(11.7)$ & $21(6.8)$ & $9(2.9)$ & $<0.001$ \\
\hline Urinary infection & $6(2.0)$ & $18(5.9)$ & $11(3.6)$ & $7(2.3)$ & 0.03 \\
\hline NIHSS at admission, median (IQR) & $4(2-8)$ & $5(3-9)$ & $4(2-8)$ & $4(2-6)$ & $<0.001$ \\
\hline TOAST subtypes, n (\%) & & & & & 0.04 \\
\hline Large artery atherosclerosis & 184(60.1) & 194(63.2) & 188(61.2) & $185(60.5)$ & \\
\hline Small artery occlusion & $74(24.2)$ & $72(23.5)$ & $87(28.3)$ & $90(29.4)$ & \\
\hline Cardioembolism & $30(9.8)$ & $22(7.2)$ & 18(5.9) & $7(2.3)$ & \\
\hline Other/undetermined & $6(2.0)$ & $9(2.9)$ & $5(1.6)$ & $12(3.9)$ & \\
\hline Undefined & $12(3.9)$ & $10(3.3)$ & $9(2.9)$ & $12(3.9)$ & \\
\hline
\end{tabular}

Abbreviations: TyG index indicates triglyceride glucose index, BMI Body mass index, IQR interquartile range, NIHSS National Institutes of Health Stroke Scale, TOAST Trial of Org 10,172 in Acute Stroke Treatment 


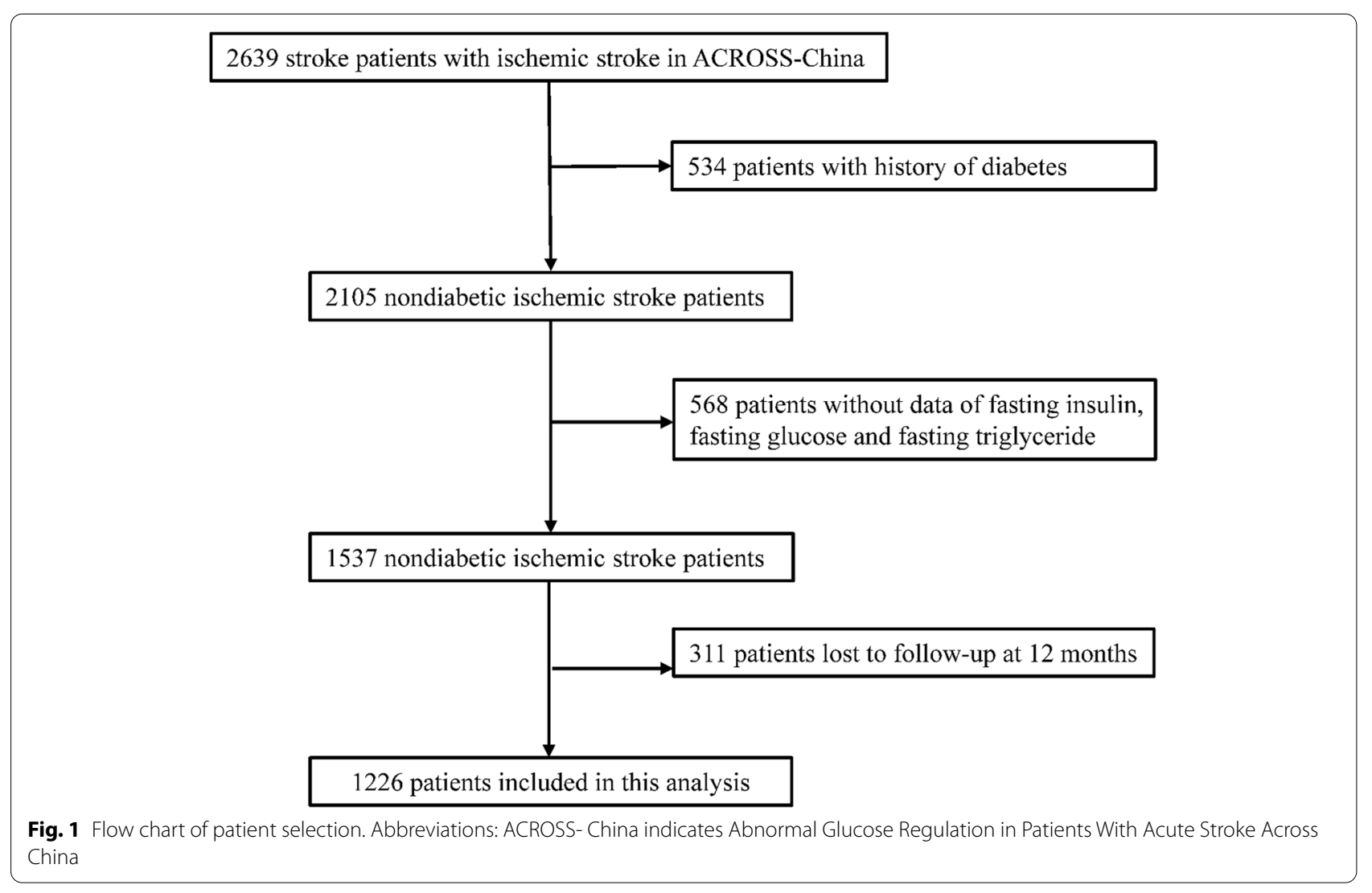

\section{Baseline characteristics}

The baseline characteristics of nondiabetic acute ischemic stroke patients according to quartiles of TyG index are shown in Table 1 . The median age was 62 years, $63.3 \%$ of them were male, and the median of TyG index was 5.8. Patients with higher TyG index were more likely to be younger; have slightly higher body mass index; have higher proportions of history of hypertension and hyperlipidemia; have lower proportions of history of atrial fibrillation; take statins during hospitalization; and have lower NIHSS at admission.

\section{Clinical outcomes according to TyG index and HOMA-IR index}

Table 2 shows the 1-year outcomes after nondiabetic acute ischemic stroke across the quartiles of TyG index. The cumulative occurrence of stroke recurrence and death was $14.5 \%$ and $9.3 \%$ at 1 -year follow up. Patients with higher TyG index quartile showed a higher incidence of stroke recurrence within 1 year (log-rank test $P=0.03)$. After adjusted for age, sex, body mass index, smoking status, medical history of hypertension, hyperlipidemia, atrial fibrillation and coronary heart disease, antihypertensive drugs, statins, intravenous alteplase, antiplatelet and anticoagulation during hospitalization, pulmonary infection and urinary infection during hospitalization, NIHSS at admission and Trial of Org 10172 in acute stroke treatment (TOAST) subtypes, patients in the third and fourth quartile of TyG index were associated with an increased risk of stroke recurrence (adjusted hazard ratio 2.04, 95\% confidence interval 1.26-3.31; adjusted hazard ratio 1.86, 95\% confidence interval 1.13-3.06). Patients with fourth quartiles of TyG index were associated with a higher mortality risk (adjusted hazard ratio, 2.91; 95\% confidence interval, $1.62-2.53 ; P<0.001)$.Similar results were observed in nondiabetic acute ischemic stroke patients without cardioembolism (Supplemental Table 2 and 3 ).

To further evaluate the predictive values of TyG index and HOMA-IR in predicting stroke recurrence and death in patients with nondiabetic acute ischemic stroke, the ROC curves and AUC regarding stroke recurrence and death were created (Fig. 2 and Fig. 3). Regarding stroke recurrence within 1 year, the AUCs (95\% confidence interval) of the ROC curve for the TyG index and HOMA-IR were $0.56(0.52-0.61)$ and 0.57 
Table 2 Adjusted hazard ratio of outcomes within 1 year according to TyG index quartiles

\begin{tabular}{|c|c|c|c|c|c|c|c|}
\hline Prognosis & TyG index & $\mathrm{n}$ & $\begin{array}{l}\text { Events, } \\
\text { n (\%) }\end{array}$ & $\begin{array}{l}\text { Model } 1 \\
\text { Unadjusted HR }(95 \% \mathrm{Cl})\end{array}$ & $P$ Value & $\begin{array}{l}\text { Model } 2^{\mathrm{a}} \\
\text { Adjusted HR }(95 \% \mathrm{Cl})\end{array}$ & $P$ Value \\
\hline \multirow[t]{5}{*}{ Stroke recurrence } & Q1 (4.31-5.48) & 298 & $31(10.4)$ & Reference & & Reference & \\
\hline & Q2 (5.48-5.81) & 290 & $37(12.8)$ & $1.38(0.83-2.29)$ & 0.21 & $1.33(0.80-2.23)$ & 0.27 \\
\hline & Q3 (5.81-6.22) & 301 & $53(17.6)$ & $1.97(1.23-3.16)$ & 0.005 & $2.04(1.26-3.31)$ & 0.004 \\
\hline & Q4 (6.22-8.17) & 296 & $51(17.2)$ & $1.86(1.15-3.00)$ & 0.01 & $1.86(1.13-3.06)$ & 0.01 \\
\hline & $P$ for trend & & & & 0.03 & & 0.03 \\
\hline \multirow[t]{5}{*}{ Death } & Q1 (4.31-5.48) & 306 & $19(6.2)$ & Reference & & Reference & \\
\hline & Q2 (5.48-5.81) & 307 & $34(11.1)$ & $1.85(1.05-3.24)$ & 0.03 & $1.94(1.09-3.48)$ & 0.03 \\
\hline & Q3 (5.81-6.22) & 307 & $23(7.5)$ & $1.14(0.62-2.10)$ & 0.69 & $1.56(0.83-2.93)$ & 0.17 \\
\hline & Q4 (6.22-8.17) & 306 & $38(12.4)$ & $2.05(1.18-3.56)$ & 0.01 & $2.91(1.62-5.23)$ & $<0.001$ \\
\hline & $P$ for trend & & & & 0.045 & & 0.002 \\
\hline
\end{tabular}

Abbreviations: TyG index indicates triglyceride glucose index, $H R$ Hazard ratio

${ }^{a}$ Adjusted for age, sex, body mass index, smoking status, medical history of hypertension, hyperlipidemia, atrial fibrillation and coronary heart disease, antihypertensive drugs, statins, intravenous alteplase, antiplatelet and anticoagulation during hospitalization, pulmonary infection and urinary infection during hospitalization, NIHSS at admission and TOAST subtypes

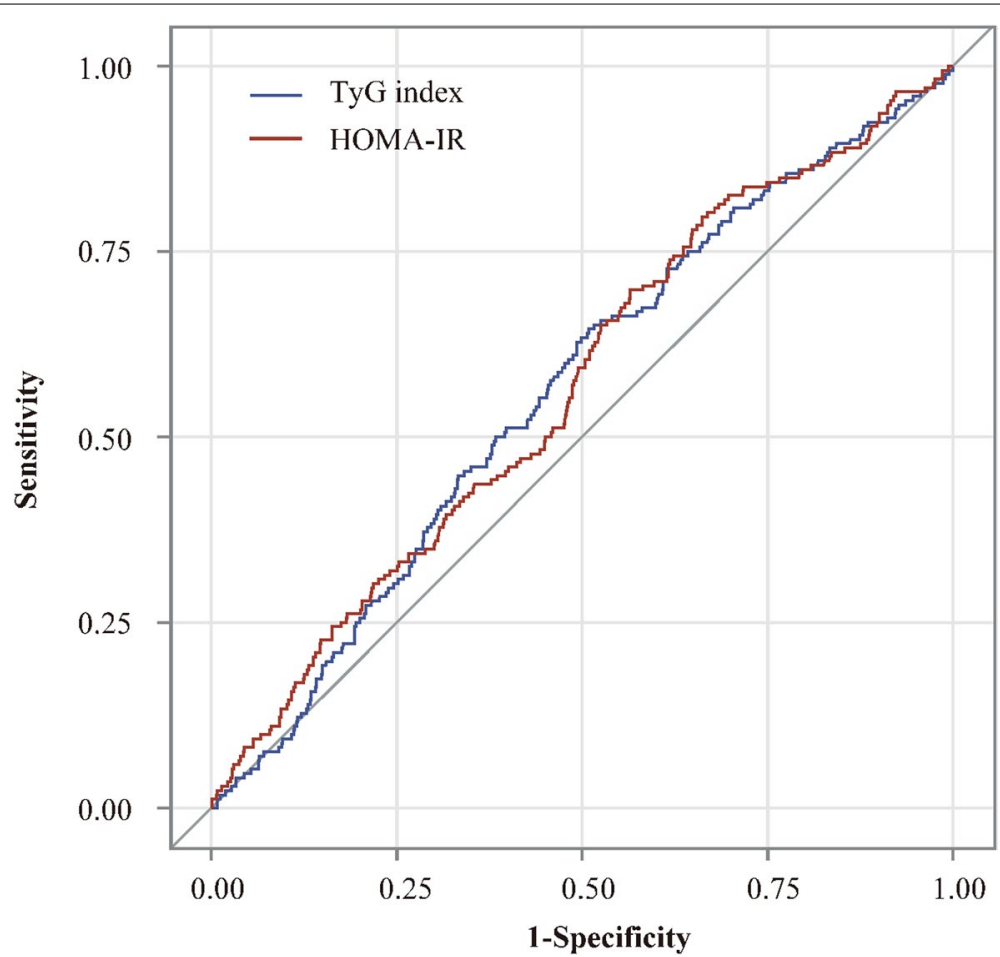

Fig. 2 Receiver operating characteristic curves of TyG index and HOMA-IR for predicting stroke recurrence within 1 year. Abbreviations: TyG index indicates triglyceride-glucose index; HOMA-IR, homeostasis model assessment of insulin resistance

(0.52-0.61), respectively $(P=0.93)$. Regarding death within 1 year, the AUCs (95\% confidence interval) of the ROC curve for the TyG index and HOMA-IR were 0.55 $(0.50-0.61)$ and $0.59(0.53-0.64)$, respectively $(P=0.32)$ (Fig. 3).

\section{Discussion}

In the current study, we found neither of TyG nor HOMA-IR can be a qualified predictor of stroke recurrence and death in nondiabetic acute ischemic stroke patients. Although patients with higher TyG index 


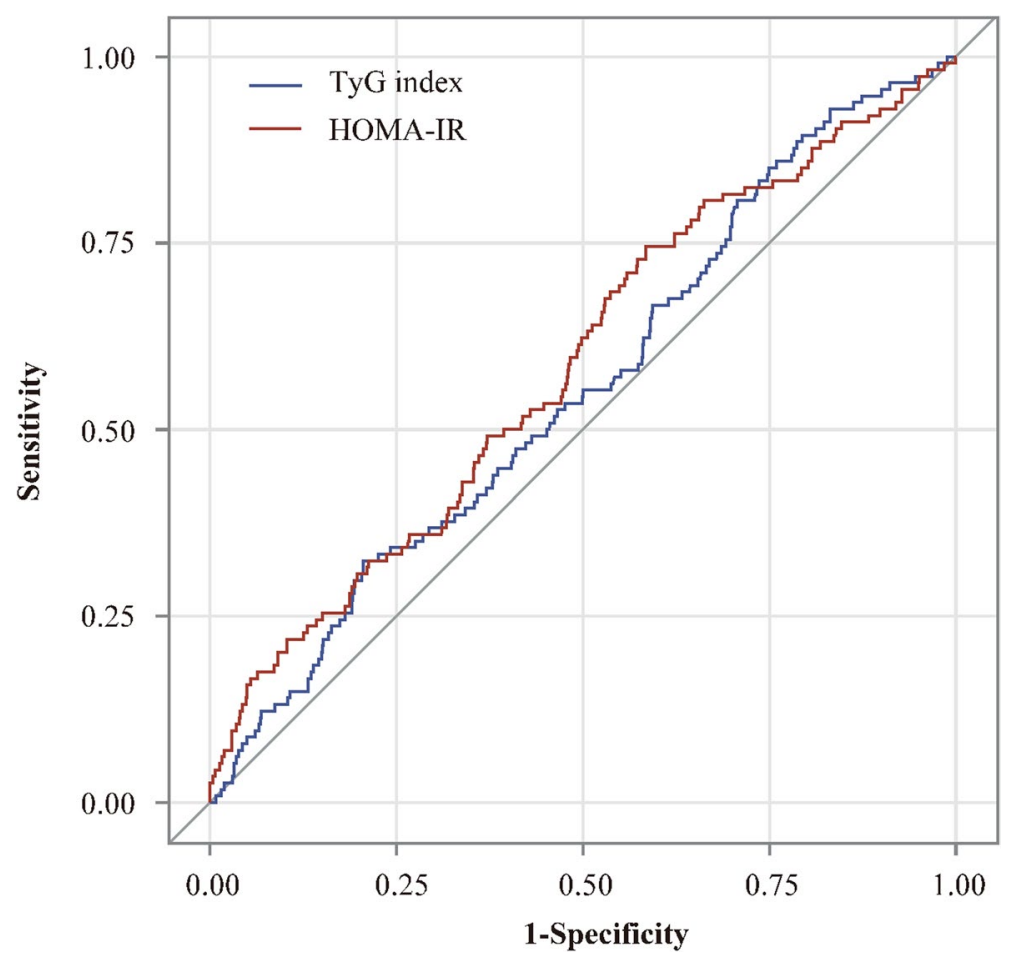

Fig. 3 Receiver operating characteristic curves of TyG index and HOMA-IR for predicting death within 1 year. Abbreviations: TyG index indicates triglyceride-glucose index; HOMA-IR, homeostasis model assessment of insulin resistance

quartile showed a higher risk of stroke recurrence and death within 1 year, from the viewpoint of clinical relevance, the curves in ROC analysis are drawn close to the diagonal line and area under curves of both TyG and HOMA-IR are relatively small (0.56 and 0.57 ), and thus their values of predicting stroke recurrence and death are judged to be low.

The rate of stroke recurrence within 1 year was $14.5 \%$, which was higher than that in other countries [15] and similar to that in China [16, 17]. China National Stroke Registry (CNSR) study conducted between September 2007 and August 2008 showed the rate of stroke recurrence within 1 year was 19.3\% [17]. Nanjing Stroke Registry demonstrated that the stroke recurrence rate within 1 year was $11.2 \%$ [16]. Several reasons might explain for the higher risk of stroke recurrence in China. Firstly, the prescription of secondary preventive medications at discharge and persistence of secondary preventive medications after stroke in China were lower than other countries [18-21]. Secondly, insulin resistance was associated with atherosclerosis [22, 23]. Intracranial artery stenoses was found commonly among stroke patients in Asian [24-26]. Intracranial stenosis or occlusion after acute cerebral ischemia was associated with higher risk of stroke recurrence [26]. The rate of stroke recurrence was $14.5 \%$ in this study, which probably reflected a high proportion of intracranial stenosis.

Glucose clamp technique is the gold standard test for quantifying insulin resistance [27], but it is limited to research purposes because of being complicated, timeconsuming and expensive. HOMA-IR based on insulin and glucose was developed as a convenient method for measuring insulin resistance, which reflected insulin resistance mainly in the liver [28]. The TyG index based on the triglyceride and glucose was even simpler to calculate, which reflected insulin resistance mainly in the skeletal muscle [29, 30].

Previous studies showed that TyG index was better associated with carotid atherosclerosis [7], nonalcoholic fatty liver disease [31], diabetes [8] and coronary artery atherosclerosis [6] than HOMA-IR. Previous studies also demonstrated that compared with the HOMA-IR, TyG index had higher sensitivity and lower specificity for recognizing insulin resistance among apparently healthy subjects [4]. However, our study showed that the predictive value of TyG index and HOMA-IR was similar in predicting stroke recurrence within 1 year. Caution is still required in our interpretations because fasting triglyceride and glucose were not measured at the same day. Further studies are 
needed to compare the predictive value of TyG index and HOMA-IR on stroke recurrence after nondiabetic acute ischemic stroke.

Although the mechanism underlying the association between the TyG index and stroke recurrence has not been clarified clearly, which may be related to insulin resistance. Insulin resistance was a syndrome that was associated with a clustering of metabolic disorders [32]. Insulin resistance was associated with obesity, diabetes mellitus, hypertension, hyperlipemia, inflammation, and atherosclerosis [32, 33], which were important risk factors of stroke recurrence [34]. Additionally, our previous studies showed insulin resistance measured by post-glucose load measures was associated with stroke recurrence [35], which was in line with TyG index in this analysis. Several potential mechanisms might account for the association between TyG index and insulin resistance. First, insulin resistance was associated with higher levels of triglyceride [36]. In return, serum levels of triglyceride was associated with insulin action in non-insulin-dependent diabetes mellitus populations [37]. Experiments showed that relative inability to store triglyceride in the subcutaneous depot might indicate a mechanism contributing to insulin resistance in the setting of obesity [38]. Second, hyperglycemia might impair insulin sensitivity [39].

There were several limitations in this study. First, among the 2105 nondiabetic acute ischemic stroke patients, 920 (43.7\%) were excluded because they were not tested for fasting glucose, triglycerides or insulin or lost to follow-up at 1 year. However, the baseline characteristics between patients included and those excluded were almost balanced. Second, compared with acute ischemic stroke patients in western countries, those in China had a higher incidence of large artery atherosclerosis. This indicated that our findings may not apply to other populations of patients with acute ischemic stroke. Third, selection bias may have existed because most of the patients was mild ischemic stroke in our study. As a standard oral glucose tolerance test was required in all the patients without prior diabetes mellitus in the ACROSS-China registry, several patients with severe clinical symptom could not complete the oral glucose tolerance test and were excluded. Fourth, the fasting triglyceride used to calculate TyG index was measured by first overnight fasting venous blood samples. The fasting insulin and fasting glucose were measured on $14 \pm 3$ days after stroke or before discharge. However, fasting glucose measured on $14 \pm 3$ days after stroke or before discharge may reduce the proportion of stress hyperglycemia [40].
Further studies about comparing the predictive value between TyG index and gold standard methods for insulin resistance are needed to confirm the results in other populations.

In conclusions, neither of TyG nor HOMA-IR can be a qualified predictor of stroke recurrence in nondiabetic acute ischemic stroke patients.

\section{Supplementary Information}

The online version contains supplementary material available at https://doi. org/10.1186/s12883-022-02588-3.

Additional file 1: Table1. Baseline characteristics of nondiabetic acute ischemic stroke patients included versus not included. Table 2. Characteristics of nondiabetic acute ischemic stroke patients without cardioembolism according to TyG index quartiles. Table 3. Adjusted hazard ratio of outcomes within 1 year according to TyG index quartiles in nondiabetic acute ischemic stroke patients without cardioembolism.

\section{Authors' contributions}

XMY and GYW: study concept and design, analysis and interpretation of data, drafting of the manuscript, and served as the equally contributing first authors of the manuscript. JJ, AXW, XLZ, QJ and HL: acquisition of data, analysis and interpretation of data. XM, XQZ and LPL: acquisition of data and revision of the manuscript. YJW and YLW: obtaining funding, study concept and design, study supervision or coordination, revision of the drafting of the manuscript. All authors read and approved the final manuscript.

\section{Funding}

The study was supported by grants from the National Natural Science Foundation of China $(81,870,905)$; the National Natural Science Foundation of China (U20A20358); Chinese Academy of Medical Sciences Innovation Fund for Medical Sciences (2019-I2M-5-029); Capital's Funds for Health Improvement and Research (2020-1-2041); the National Natural Science Foundation of China (No. 81825007); Beijing Outstanding Young Scientist Program (No. BJJWZYJH01201910025030); Youth Beijing Scholar Program (No.010).

\section{Availability of data and materials}

The datasets during and/or analysed during the current study available from the corresponding author on reasonable request.

\section{Declarations}

\section{Ethics approval and consent to participate}

The study complied with the Declaration of Helsinki, and protocol was approved by the IRB of Beijing Tiantan Hospital, Capital Medical University. All patients or their legal representatives provided written informed consent.

\section{Consent for publication}

Not applicable

\section{Competing interests}

The author(s) declared no potential conflicts of interest with respect to the research, authorship, and/or publication of this article.

\section{Author details}

${ }^{1}$ Department of Neurology, Beijing Tiantan Hospital, Capital Medical University, No.119, South 4th Ring West Road, Fengtai District, Beijing 100070, China. ${ }^{2}$ China National Clinical Research Center for Neurological Diseases, Beijing, China. ${ }^{3}$ Department of Neurology, Beijing Jishuitan Hospital, Beijing, China. ${ }^{4}$ Advanced Innovation Center for Human Brain Protection, Capital Medical University, Beijing, China. 
Received: 18 June 2021 Accepted: 31 January 2022

Published online: 08 March 2022

\section{References}

1. Bonora E, Kiechl S, Willeit J, Oberhollenzer F, Egger G, Targher G, Alberiche M, Bonadonna RC, Muggeo M. Prevalence of insulin resistance in metabolic disorders: the bruneck study. Diabetes. 1998:47:1643-9.

2. Kernan WN, Inzucchi SE, Viscoli CM, Brass LM, Bravata DM, Shulman GI, MCVeety JC, Horwitz RI. Impaired insulin sensitivity among nondiabetic patients with a recent TIA or ischemic stroke. Neurology. 2003:60:1447-51

3. Jing J, Pan Y, Zhao X, Zheng H, Jia Q, Mi D, Chen W, Li H, Liu L, Wang $C$, et al. Insulin resistance and prognosis of nondiabetic patients with ischemic stroke: The ACROSS-China study (abnormal glucose regulation in patients with acute stroke across China). Stroke. 2017;48:887-93.

4. Simental-Mendía LE, Rodríguez-Morán M, Guerrero-Romero F. The product of fasting glucose and triglycerides as surrogate for identifying insulin resistance in apparently healthy subjects. Metab Syndr Relat Disord. 2008;6:299-304

5. Guerrero-Romero F, Villalobos-Molina R, Jiménez-Flores JR, Simental-Mendia LE, Méndez-Cruz R, Murguía-Romero M, Rodríguez-Morán M. Fasting triglycerides and glucose index as a diagnostic test for insulin resistance in young adults. Arch Med Res. 2016;47:382-7.

6. Kim MK, Ahn CW, Kang S, Nam JS, Kim KR, Park JS. Relationship between the triglyceride glucose index and coronary artery calcification in Korean adults. Cardiovasc Diabetol. 2017;16:108.

7. Irace C, Carallo C, Scavelli FB, De Franceschi MS, Esposito T, Tripolino C, Gnasso A. Markers of insulin resistance and carotid atherosclerosis. A comparison of the homeostasis model assessment and triglyceride glucose index. Int J Clin Pract. 2013;67:665-72.

8. Lee $\mathrm{SH}$, Kwon HS, Park YM, Ha HS, Jeong SH, Yang HK, Lee JH, Yim HW, Kang MI, Lee WC, et al. Predicting the development of diabetes using the product of triglycerides and glucose: the Chungju Metabolic Disease Cohort (CMC) study. PLoS ONE. 2014;9:e90430.

9. Tohidi M, Baghbani-Oskouei A, Ahanchi NS, Azizi F, Hadaegh F. Fasting plasma glucose is a stronger predictor of diabetes than triglycerideglucose index, triglycerides/high-density lipoprotein cholesterol, and homeostasis model assessment of insulin resistance: tehran lipid and glucose study. Acta Diabetol. 2018;55:1067-74.

10. Jia Q, Zheng H, Zhao X, Wang C, Liu G, Wang Y, Liu L, Li H, Zhong L, Wang $Y$, et al. Abnormal glucose regulation in patients with acute stroke across China: prevalence and baseline patient characteristics. Stroke. 2012;43:650-7.

11. Stroke--1989. Recommendations on stroke prevention, diagnosis, and therapy. Report of the WHO Task Force on Stroke and other Cerebrovascular Disorders. Stroke. 1989;20:1407-1431.

12. Adams HP Jr, Bendixen BH, Kappelle LJ, Biller J, Love BB, Gordon DL, Marsh EE 3rd. Classification of subtype of acute ischemic stroke. Definitions for use in a multicenter clinical trial. TOAST. Trial of Org 10172 in Acute Stroke Treatment. Stroke. 1993;24:35-41.

13. Matthews DR, Hosker JP, Rudenski AS, Naylor BA, Treacher DF, Turner RC. Homeostasis model assessment: insulin resistance and beta-cell function from fasting plasma glucose and insulin concentrations in man. Diabetologia. 1985;28:412-9.

14. Pan Y, Chen W, Jing J, Zheng H, Jia Q, Li H, Zhao X, Liu L, Wang Y, He Y, et al. Pancreatic beta-cell function and prognosis of nondiabetic patients with ischemic stroke. Stroke. 2017:48:2999-3005.

15. Feng W, Hendry RM, Adams RJ. Risk of recurrent stroke, myocardial infarction, or death in hospitalized stroke patients. Neurology. 2010:74:588-93.

16. Xu G, Liu X, Wu W, Zhang R, Yin Q. Recurrence after ischemic stroke in chinese patients: impact of uncontrolled modifiable risk factors. Cerebrovasc Dis. 2007;23:117-20

17. Deng $Y X$, Wang $Y L$, Gao BQ, Wang $C X$, Zhao $X Q$, Liu LP, Wang $A X$, Zhou Y, Liu GF, Du WL, et al. Age differences in clinical characteristics, health care, and outcomes after ischemic stroke in China. CNS Neurosci Ther. 2012;18:819-26.

18. Ji R, Liu G, Shen H, Wang Y, Li H, Peterson E, Wang Y. Persistence of secondary prevention medications after acute ischemic stroke or transient ischemic attack in Chinese population: data from China National Stroke Registry. Neurol Res. 2013;35:29-36.

19. Zhang J, Gong Y, Zhao Y, Jiang N, Wang J, Yin X. Post-stroke medication adherence and persistence rates: a meta-analysis of observational studies. J Neurol. 2019;268(6):2090.

20. Bushnell CD, Olson DM, Zhao X, Pan W, Zimmer LO, Goldstein LB, Alberts MJ, Fagan SC, Fonarow GC, Johnston SC, et al. Secondary preventive medication persistence and adherence 1 year after stroke. Neurology. 2011;77:1182-90.

21. Qin H, Chen Y, Liu G, Turnbull I, Zhang R, Li Z, Wang Y, Liu L, Zhao X, Chen $Z$, et al. Management characteristics and prognosis after stroke in China: findings from a large nationwide stroke registry. Stroke Vasc Neurol. 2020;6:1.

22. Pané A, Conget I, Boswell L, Ruiz S, Viñals C, Perea V, Giménez M, Cofán M, Blanco J, Vinagre I, et al. Insulin resistance is associated with preclinical carotid atherosclerosis in patients with type 1 diabetes. Diabetes Metab Res Rev. 2020;7:e3323.

23. Di Pino A, DeFronzo RA. Insulin resistance and atherosclerosis: implications for insulin-sensitizing agents. Endocr Rev. 2019;40:1447-67.

24. Sacco RL, Kargman DE, Gu Q, Zamanillo MC. Race-ethnicity and determinants of intracranial atherosclerotic cerebral infarction. The northern manhattan stroke study. Stroke. 1995;26:14-20.

25. Wong LK. Global burden of intracranial atherosclerosis. Int J Stroke. 2006:1:158-9.

26. Wang Y, Zhao X, Liu L, Soo YO, Pu Y, Pan Y, Wang Y, Zou X, Leung TW, Cai Y, et al. Prevalence and outcomes of symptomatic intracranial large artery stenoses and occlusions in China: the Chinese Intracranial Atherosclerosis (CICAS) Study. Stroke. 2014;45:663-9.

27. DeFronzo RA, Tobin JD, Andres R. Glucose clamp technique: a method for quantifying insulin secretion and resistance. Am J Physiol. 1979:237:E214-223.

28. Tripathy D, Almgren P, Tuomi T, Groop L. Contribution of insulin-stimulated glucose uptake and basal hepatic insulin sensitivity to surrogate measures of insulin sensitivity. Diabetes Care. 2004;27:2204-10.

29. Kelley DE, Goodpaster BH. Skeletal muscle triglyceride. An aspect of regional adiposity and insulin resistance. Diabetes Care. 2001;24:933-41.

30. Riboldi BP, Luft VC, de Castilhos CD, de Cardoso LO, Schmidt MI, Barreto SM, de Sander MF, Alvim SM, Duncan BB. Glucose and triglyceride excursions following a standardized meal in individuals with diabetes: ELSABrasil study. Cardiovasc Diabetol. 2015:14:21.

31. Lee SB, Kim MK, Kang S, Park K, Kim JH, Baik SJ, Nam JS, Ahn CW, Park JS. Triglyceride glucose index is superior to the homeostasis model assessment of insulin resistance for predicting nonalcoholic fatty liver disease in Korean adults. Endocrinol Metab (Seoul). 2019:34:179-86.

32. DeFronzo RA, Ferrannini E. Insulin resistance. A multifaceted syndrome responsible for NIDDM, obesity, hypertension, dyslipidemia, and atherosclerotic cardiovascular disease. Diabetes Care. 1991;14:173-94.

33. Kernan WN, Inzucchi SE, Viscoli CM, Brass LM, Bravata DM, Horwitz RI. Insulin resistance and risk for stroke. Neurology. 2002;59:809-15.

34. Kernan WN, Ovbiagele B, Black HR, Bravata DM, Chimowitz MI, Ezekowitz MD, Fang MC, Fisher M, Furie KL, Heck DV, et al. Guidelines for the prevention of stroke in patients with stroke and transient ischemic attack: a guideline for healthcare professionals from the American Heart Association/American Stroke Association. Stroke. 2014;45:2160-236.

35. Pan Y, Jing J, Chen W, Zheng H, Jia Q, Mi D, Li H, Zhao X, Liu L, Wang $\mathrm{C}$, et al. Post-Glucose load measures of insulin resistance and prognosis of nondiabetic patients with ischemic stroke. J Am Heart Assoc. 2017;6:e004990.

36. Abbasi F, McLaughlin T, Lamendola C, Yeni-Komshian H, Tanaka A, Wang T, Nakajima K, Reaven GM. Fasting remnant lipoprotein cholesterol and triglyceride concentrations are elevated in nondiabetic, insulin-resistant, female volunteers. J Clin Endocrinol Metab. 1999;84:3903-6.

37. Taniguchi A, Fukushima M, Sakai M, Kataoka K, Nagata I, Doi K, Arakawa $\mathrm{H}$, Nagasaka S, Tokuyama K, Nakai Y. The role of the body mass index and triglyceride levels in identifying insulin-sensitive and insulin-resistant variants in Japanese non-insulin-dependent diabetic patients. Metabolism. 2000;49:1001-5.

38. Allister CA, Liu LF, Lamendola CA, Craig CM, Cushman SW, Hellerstein MK, McLaughlin TL. In vivo $2 \mathrm{H} 2 \mathrm{O}$ administration reveals impaired triglyceride storage in adipose tissue of insulin-resistant humans. J Lipid Res. 2015:56:435-9. 
39. Yki-Järvinen H. Glucose toxicity. Endocr Rev. 1992;13:415-31.

40. Gray CS, Scott JF, French JM, Alberti KG, O'Connell JE. Prevalence and prediction of unrecognised diabetes mellitus and impaired glucose tolerance following acute stroke. Age Ageing. 2004;33:71-7.

\section{Publisher's Note}

Springer Nature remains neutral with regard to jurisdictional claims in published maps and institutional affiliations.

- fast, convenient online submission

- thorough peer review by experienced researchers in your field

- rapid publication on acceptance

- support for research data, including large and complex data types

- gold Open Access which fosters wider collaboration and increased citations

- maximum visibility for your research: over $100 \mathrm{M}$ website views per year

At BMC, research is always in progress.

Learn more biomedcentral.com/submissions 